VOL. $42(1990) \quad[399-406]$

\title{
ON A GENERAL NONLINEAR VARIATIONAL INEQUALITY
}

\author{
Ramendra Krishna Bose
}

\begin{abstract}
Variational inequality theory provides techniques for solving a variety of applied problems in science and engineering. Recently Noor considered some interesting general nonlinear and linear variational inequalities in a series of papers and proved the existence and uniqueness of solutions by a fixed point technique developed by Glowinski, Lions and Tremolieres and also by a fixed point technique of Lions and Stampacchia. But there are several inaccuracies in his proofs and here they have been removed and correct formulation of the theorems are stated and proved and relationships are clearly shown. The existence of solution necessitates an additional condition in one case, and less condition in the other, but uniqueness can be proved without the condition that the operator be antimonotone.
\end{abstract}

\section{INTRODUCTION}

Variational inequality theory provides techniques to solve a variety of applied problems in science and engineering. For details, one can refer to Duvaut and Lions [3], Glowinski, Lions and Tremolieres [4], Crank [2], and Baiocchi and Capelo [1]. Recently Noor [5] considered some general nonlinear variational inequalities and proved the existence of a unique solution by a fixed point technique of Glowinski, Lions and Tremolieres [4] under certain conditions. Previously known classes of variational inequalities, originally studied by Duvaut and Lions [3], are special cases of this general inequality. Earlier Noor [6] considered a less generalised variational inequality related with a Signorini problem in which also he used the similar techniques to prove the existence of a unique solution under certain conditions. Noor [9] has considered the same general variational inequality again with some variation of conditions and proved the existence of a unique solution by a fixed point technique of Lions and Stampacchia [7] (or refer to Baiocchi and Capelo [1], p.26 - the second proof). Earlier Noor [8] considered a less general version of this variational inequality.

There are certain inaccuracies in the proofs given by Noor $[5,6,8,9]$, and the object of this note is to prove the theorems under suitable conditions which necessitates certain deletion and addition of conditions. The specific differences between the results obtained by these two fixed point techniques are established. Naturally this will lead to the required modification in the results and and examples discussed by Noor [9].

Received 30 November, 1989.

Copyright Clearance Centre, Inc. Serial-fee code: 0004-9729/90 SA2.00+0.00. 


\section{Preliminaries and Notation.}

Let $H$ be a Hilbert space on the reals with its dual $H^{\prime}$, whose norm and inner product are denoted by $\|\cdot\|$ and $(.,$.$) , respectively. We denote the pairing between H^{\prime}$ and $H$ by $(.,$.$) . We have (f, u)=(\Lambda f, u)$ for all $f \in B^{\prime}$ and $u \in H$, where $\Lambda$ is the canonical isomorphism from $H^{\prime}$ onto $H$. Also $\|\Lambda\|=\left\|\Lambda^{-1}\right\|=1$.

Let $K$ be a closed convex set in $H$ and $a(.,):. H \times H \rightarrow R$ be a coercive continuous bilinear form on $H$. That is, there exist constants $\alpha>0$, and $\beta>0$ such that the bilinear form satisfies the following:

$$
a(v, v) \geqslant \alpha\|v\|^{2}
$$

and

$$
a(u, v) \leqslant \beta\|u\|\|v\| \quad \text { for all } \quad u, v \in H .
$$

It follows from (1) and (2) that $\alpha \leqslant \beta$. Let $j: H \rightarrow R \cup\{\infty\} \cup\{-\infty\}$ be a functional which is convex, lower semi-continuous and proper (that is, $j(v)>-\infty$ for all $v \in$ $H$, and $j \not \equiv \infty)$.

Moreover let the form $b(.,):. H \times H \rightarrow R$ which is not differentiable, satisfy the following:

(i) $b(u, v)$ is linear in the first argument,

(ii) $b(u, v)$ is bounded, that is, there exists a constant $\gamma>0$ such that

$$
|b(u, v)| \leqslant \gamma\|u\|\|v\| \text { for all } u, v \in H,
$$

(iii) $b(u, v)-b(u, w) \leqslant b(u, v-w)$ for all $u, v$, and $w \in H$, and

(iv) for a fixed $u, b(u, v)$, that is, $b(u,$.$) is a convex functional.$

When $b(u, v)$ is linear in the second argument, conditions (iii) and (iv) are satisfied.

A non-linear operator $A: H \rightarrow H^{\prime}$ is called Lipschitz continuous if there exists a constant $\xi>0$ such that

$$
\|A u-A v\| \leqslant \xi\|u-v\|, \quad \text { for all } \quad u, v \in H,
$$

and is called antimonotone if

$$
\langle A u-A v, u-v\rangle \leqslant 0 \text { for all } u, v \in K .
$$

As $a(u, v)$ is a continuous bilinear form on $H$, by the Riesz-Frechet representation theorem, we have

$$
a(u, v)=\langle T u, v\rangle
$$

for all $v \in H$ where $T: H \rightarrow H^{\prime}$. Furthermore $\|T\| \leqslant \beta$. 


\section{MAIN RESULT.}

We first state the theorems proved by Noor $([5,6])$.

Theorem A. (Noor [6]) Let $a(u, v)$ be a coercive continuous bilinear form and $j(v)$ be a convex, lower semi-continuous proper functional. If $A: K \rightarrow H^{\prime}$ is a Lipschitz continuous antimonotone operator and condition

$$
\gamma<\alpha
$$

holds, then there exists a unique solution $u \in K$ such that

$$
a(u, v-u)+j(v)-j(u) \geqslant\langle A(u), v-u\rangle, \quad \text { for all } v \in K .
$$

ThEOREM B. (Noor [5]) Let $a(u, v)$ be a coercive continuous bilinear form and $b(u, v)$ satisfy the conditions (i)-(iii). If $A: K \rightarrow H^{\prime}$ is a Lipschitz continuous antimonotone operator and condition

$$
\gamma+\xi<\alpha
$$

holds, then there exists a unique solution $u \in K$ such that

$$
a(u, v-u)+b(u, v)-b(u, u) \geqslant\langle A(u), v-u\rangle \quad \text { for all } v \in K .
$$

The theorems we want to prove are stated below.

THEOREM 1. Let $a(u, v)$ be a coercive continuous bilinear form and $j(v)$ be a convex, lower semi-continuous proper functional. If $A: K \rightarrow H^{\prime}$ is a Lipschitz continuous operator and condition $(N)$ holds, then there exists a unique solution $u \in K$ such that

$$
a(u, v-u)+j(v)-j(u) \geqslant\langle A(u), v-u\rangle,
$$

for all $v \in K$ (that is, $A$ need not be antimonotone).

THEOREM 2. Let $a(u, v)$ be a coercive continuous bilinear form and $b(u, v)$ satisfy the conditions (i)-(iv). If $A: K \rightarrow H^{\prime}$ is Lipschitz continuous operator and condition $\left(N^{*}\right)$ holds, then there exists a unique solution $u \in K$ such that

$$
a(u, v-u)+b(u, v)-b(u, u) \geqslant\langle A(u), v-u\rangle
$$

for all $v \in K$ (that is, $A$ need not be antimonotone but $b(.,$.$) has to satisfy the$ additional condition (iv) for the existence and uniqueness of the auxiliary problem discussed below). 
REMARK 1. If the dependence of the form $b(u, v)$ is restricted to its second argument only, that is, $b(u, v)=j(v)$, the variational inequality (7) reduces to the variational inequality (6) with some stronger conditions on $j$. We give the proof of Theorem 2 only. Theorem 1 can be proved in a similar fashion.

Proof of Theorem 2: We prove the uniqueness of solution first. Let $u_{1}, u_{2} \in K$ be two solutions of (7). Then

$$
a\left(u_{1}, v-u_{1}\right)+b\left(u_{1}, v\right)-b\left(u_{1}, u_{1}\right) \geqslant\left\langle A\left(u_{1}\right), v-u_{1}\right\rangle
$$

for all $v \in K$ and

$$
a\left(u_{2}, v-u_{2}\right)+b\left(u_{2}, v\right)-b\left(u_{2}, u_{2}\right) \geqslant\left\langle A\left(u_{2}\right), v-u_{2}\right\rangle
$$

for all $v \in K$.

Under stated conditions (in case $b(u, v)=j(v)$ refer to Glowinski et al. [4], p.543), we can take $v=u_{2}$ in (8) and $v=u_{1}$ in (9), and adding these inequalities and using condition (iii), we get

$$
a\left(u_{1}-u_{2}, u_{1}-u_{2}\right) \leqslant\left(\Lambda\left(A\left(u_{1}\right)-A\left(u_{2}\right)\right), u_{1}-u_{2}\right)+b\left(u_{1}-u_{2}, u_{2}-u_{1}\right) .
$$

Using (1), (3), and condition (ii) of $b(.,$.$) in (10) we have$

$$
\alpha\left\|u_{1}-u_{2}\right\|^{2} \leqslant \xi\left\|u_{1}-u_{2}\right\|^{2}+\gamma\left\|u_{1}-u_{2}\right\|^{2}
$$

That is $(\alpha-\xi-\gamma)\left\|u_{1}-u_{2}\right\|^{2} \leqslant 0$. Since $\alpha>\xi+\gamma$ by condition $\left(N^{*}\right)$, we have $u_{1}=u_{2}$.

For existence, we have to consider the following auxiliary problem. For each $u \in K$ and $\rho>0$, we consider the auxiliary problem of finding $w \in K$ satisfying the variational inequality problem:

Find $w \in K$ such that

$$
\begin{aligned}
(w, v-w) & +\rho b(u, v)-\rho b(u, w) \geqslant(u, v-w) \\
& +\rho(A(u), v-w\rangle-\rho a(u, v-w)
\end{aligned}
$$

for all $v \in K$.

For a fixed $u$, this is the problem $\left(\Pi_{\rho}(u)\right)$ of Glowinski, Lions and Tremolieres [4] (Appendix 1, Sec. 2, p.545, Lemma 2.1). This problem admits one and only one solution when the conditions of Theorem 2 are satisfied (of course $b$ need satisfy conditions (ii) to (iv) only).

Let $w_{1}, w_{2}$ be two solutions of (11) related to $u_{1}, u_{2} \in K$, respectively. We can prove the existence of a solution of (7) if we show that the mapping $u \rightarrow w \quad(w=S u)$ has a fixed point in $K$. For this we show that $S: K \rightarrow K$ satisfies the condition

$$
\left\|S u_{2}-S u_{1}\right\| \leqslant \theta\left\|u_{2}-u_{1}\right\| \text { with } 0 \leqslant \theta<1,
$$


where $\theta$ is independent of $u_{1}$ and $u_{2}$.

Taking $v=w_{2}$ (respectively $\left.w_{1}\right)$ in (11) related to $u_{1}$ (respectively $u_{2}$ ), we get

$$
\begin{aligned}
\left(w_{1}, w_{2}-w_{1}\right) & +\rho b\left(u_{1}, w_{2}\right)-\rho\left(u_{1}, w_{1}\right) \geqslant\left(u_{1}, w_{2}-w_{1}\right) \\
& +\rho\left\langle A\left(u_{1}\right), w_{2}-w_{1}\right\rangle-\rho a\left(u_{1}, w_{2}-w_{1}\right) \\
\left(w_{2}, w_{1}-w_{2}\right) & +\rho b\left(u_{2}, w_{1}\right)-\rho b\left(u_{2}, w_{2}\right) \geqslant\left(u_{2}, w_{1}-w_{2}\right) \\
& +\rho\left\langle A\left(u_{2}\right), w_{1}-w_{2}\right\rangle-\rho a\left(u_{2}, w_{1}-w_{2}\right) .
\end{aligned}
$$

Adding (12) and (13) and using (5) we get, after changing sign,

$$
\begin{gathered}
\left\|w_{2}-w_{1}\right\|^{2} \leqslant \rho b\left(u_{1}-u_{2}, w_{2}-w_{1}\right) \\
+\left(u_{2}-u_{1}-\rho \Lambda\left(T u_{2}-T u_{1}\right), w_{2}-w_{1}\right)-\rho\left(\Lambda\left(A\left(u_{1}\right)-A\left(u_{2}\right)\right), w_{2}-w_{1}\right),
\end{gathered}
$$

that is

$$
\left\|w_{2}-w_{1}\right\| \leqslant\left[\sqrt{1-2 \alpha \rho+\alpha^{2} \beta^{2}}+\rho(\gamma+\xi)\right]\left\|u_{2}-u_{1}\right\|
$$

(Glowinski, Lions, and Tremolieres [4], p.16). Here

where

$$
\begin{gathered}
\theta=\sqrt{1-2 \alpha \rho+\rho^{2} \beta^{2}}+\rho(\gamma+\xi)<1 \\
0<\rho<2 \frac{\alpha-\gamma-\xi}{\beta^{2}-(\gamma+\xi)^{2}} \text { and } \rho<\frac{1}{\gamma+\xi}
\end{gathered}
$$

(by condition $\left(\mathrm{N}^{*}\right)$ we have $\left.\gamma+\xi<\alpha \leqslant \beta\right)$.

Remark 2. When $b(u, v)=j(v)$, Theorem 2 gives Theorem 1 with a little stronger condition on $j(v)$ (continuity instead of lower semi-continuity and range of $j(v)$ restricted to reals). But Theorem 1 can be proved exactly in the same manner under the stated conditions.

Now we discuss the solution of these general variational inequalities by the fixed point technique of Lions and Stampacchia [7]. Noor proved the following two theorems.

THEOREM C. (Noor [9]) Let $a(u, v)$ be a coercive continuous bilinear form and $b(u, v)$ satisfy the conditions (i) and (ii), and let $b(u, v)$ be sublinear in the second argument, that is,

$$
b(u, u-v) \leqslant b(u, u)-b(u, v) \quad \text { for all } \quad u, v \in H .
$$

If the condition $(N)$ holds, then there exists a unique $u \in K$ such that, for a given $f \in \boldsymbol{H}^{\prime}$,

$$
a(u, v-u)+b(u, v)-b(u, u) \geqslant\langle f, v-u\rangle \text { for all } v \in K \text {. }
$$


ThEOREM D. (Noor [8]) Let $a(u, v)$ be a coercive continuous bilinear form and $b(u, v)$ satisfy conditions (i) and (ii) and also the following: It is either convex or linear in the second argument and

$$
\begin{gathered}
|b(u, v)-b(u, w)| \leqslant b(u, v-w), \\
b(u, v \pm w) \leqslant b(u, v)+b(u, w) \quad \text { for every } u, v, w \in H
\end{gathered}
$$

If the operator $A$ is antimonotone and Lipschitz continuous and condition $\left(N^{*}\right)$ holds, then there exists a unique solution $u \in K$ such that

$$
a(u, v-u)+b(u, v)-b(u, u) \geqslant\langle A(u), v-u\rangle \quad \text { for all } \quad v \in K .
$$

The theorems we want to prove are stated below.

THEOREM 3. Let $a(u, v)$ be a coercive continuous bilinear form and $b(u, v)$ satisfy conditions (i) and (ii) and linearity in the second argument. If condition $(N)$ holds, then there exists a unique $u \in K$ such that, for a given $f \in H^{\prime}$,

$$
a(u, v-u)+b(u, v)-b(u, u) \geqslant\langle f, v-u\rangle \quad \text { for all } \quad v \in K .
$$

(That is, we need a stronger condition on $b(u, v)$.)

THEOREM 4. Let $a(u, v)$ be a coercive continuous bilinear form and $b(u, v)$ be a continuous bilinear form. If the non-linear operator is Lipschitz continuous and condition $\left(N^{*}\right)$ holds, then there exists a unique $u \in K$ such that

$$
a(u, v-u)+b(u, v)-b(u, u) \geqslant\langle A(u), v-u\rangle \quad \text { for all } \quad v \in K .
$$

(Neither the convexity of $b(u, v)$ in the second argument nor the antimonotonicity of the operator $A$ is needed and linearity of $b(u, v)$ in the second argument is sufficient.)

REMARK 3. Theorem 4 is a corollary of Theorem 2. Theorem 3 is a corollary of Thoerem 4 when $A(u) \in H^{\prime}$ is independent of $u$ and equals $f \in H^{\prime}$. The proof of Theorem 4 can be given in the same manner as in Noor [9] under the stated conditions. (A short sketch is given below.) Thus we conclude that the fixed point technique of Glowinski et al gives us a better result compared to the fixed point technique of Lions and Stampacchia.

We need the following lemmas (refer to Baiocchi and Capelo [1], p.18) to prove Theorem 4 by the fixed point technique of Lions and Stampacchia.

Lemma 1. Let $M$ be a convex subset of $H$. Then, given $z \in H$, we have $u=P_{M} z$ (projection of $z$ onto $M$ ) if and only if

$$
u \in M:(u-z, v-u) \geqslant 0 \text { for all } v \in M .
$$


Lemma 2. The projection operator $P_{M}$ is non-expansive, that is,

$$
\left\|P_{M} u-P_{M} v\right\| \leqslant\|u-v\| \quad \text { for all } \quad u, v \in H .
$$

Proof of ThEOREM 4: The uniqueness can be shown as in the proof of Theorem 2. For existence, for a fixed $\rho>0$ and $u \in H$, we define $\phi(u) \in H^{\prime}$ by

$$
\langle\phi(u), v\rangle=(u, v)-\rho a(u, v)-\rho b(u, v)+\rho\langle A(u), v\rangle \quad \text { for all } \quad v \in H .
$$

By Lemma 1, there exists $w \in K$ such that

$$
(w, v-w) \geqslant\langle\phi(u), v-w\rangle \quad \text { for all } \quad v \in K
$$

and $w$ is given by $w=P_{K} \Lambda \phi(u)=T u$ and this defines a mapping from $H$ into $K$. Also by Lemma 2, it can be shown (as in the proof of Theorem 2) that

$$
\begin{aligned}
\|T u-T v\| & \leqslant\|\Lambda \phi(u)-\Lambda \phi(v)\| \\
& \leqslant\|\phi(u)-\phi(v)\| \leqslant \theta\|u-v\| \text { for all } u, v \in H .
\end{aligned}
$$

Here $\theta$ is same as given in (14). Thus $T$ is a contraction mapping from $K$ into itself and it has a fixed point, say $u$. Then by (16) we have

$$
(u, v-u) \geqslant\langle\phi(u), v-u\rangle \quad \text { for all } \quad v \in K .
$$

This implies that

$$
a(u, v-u)+b(u, v)-b(u, u) \geqslant\langle A(u), v-u\rangle \quad \text { for all } \quad v \in K .
$$

\section{REFERENCES}

[1] C. Baiocchi and A. Capelo, Variational and quasi-variational inequalities (Wiley, New York, London, 1984).

[2] J. Crank, Free and moving boundary problems (Oxford University Press, United Kingdom, 1984).

[3] G. Duvaut and J. Lions, Les inequations en mechanique et en physique (Dunod, Paris, 1972).

[4] R. Glowinski, J. Lions, and R. Tremolieres, Numerical analysis of variational inequalities (North Holland, Amsterdam, 1981).

[5] M. Aslam Noor, 'General nonlinear variational inequalities', J. Math. Anal. Appl. 126 (1987), 78-84. 
[6] M. Aslam Noor, 'Variational inequalities related with a Signorini problem', C.R. Math. Rep. Acad. Sci. Canada 7 (1985), 267-272.

[7] J. Lions and G. Stampacchia, 'Variational inequalities', Comm. Pure Appl. Math. 20 (1967), 493-519.

[8] M. Aslam Noor, 'Variational inequalities for a class of contact problems with friction', C.R. Math. Rep. Acad. Sci. Canada 5 (1983), 127-132.

[9] M. Aslam Noor, 'On a class of variational inequalities', J. Math. Anal. Appl. 128 (1987), 138-155.

Department of Mathematics and Computer Science

State University of New York

College at Fredonia

Fredonia NY 14063

United States of America 